\title{
Index verborum memorabilium
}

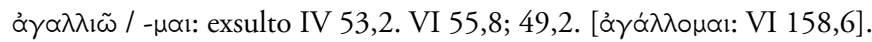

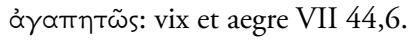

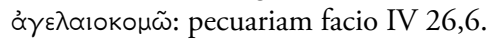

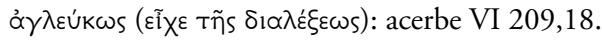

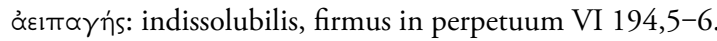

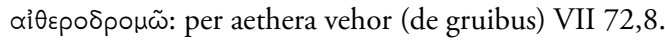

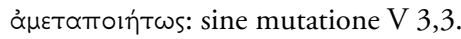

ớ ${ }^{\prime} \pi \lambda \alpha \sigma ı s:$ imaginatio VI 29,6.

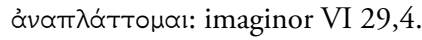

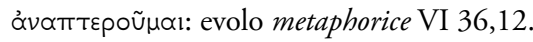

ơoóppous: fluxus in altum (sucorum corporis) VI 128,8.

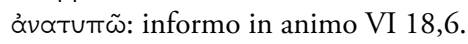

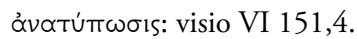

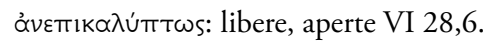

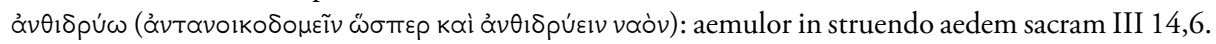

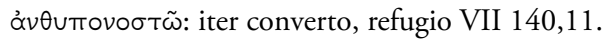

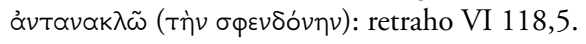

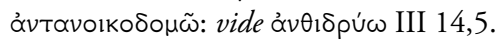

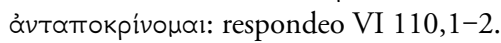

ơ $v т \varepsilon \dot{\sigma} \sigma \varepsilon ı \mu$ : in locum alicuius ingredior $\mathrm{V} 1,8$.

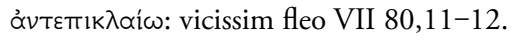

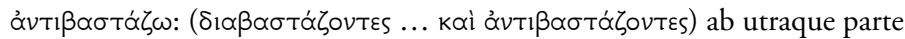

premens sustineo VI 129,6.

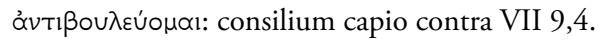

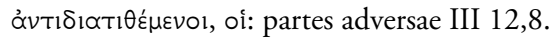

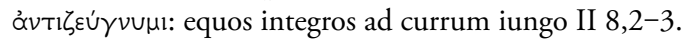

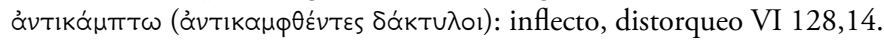

$\alpha \dot{\alpha} v i^{\prime} \lambda \eta \psi 1 s:$ contraprehensio qua athleta adversarium corripit VI 190,4.

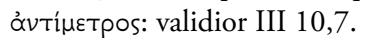

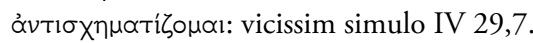

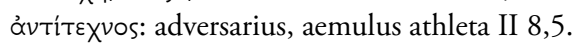

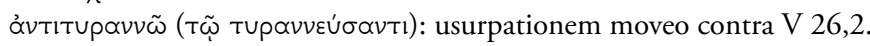

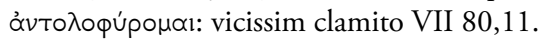

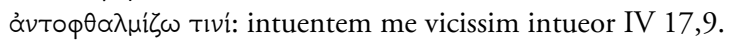

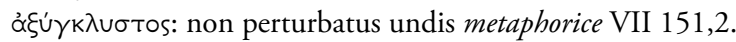

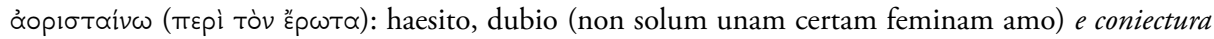

VI 151,3.

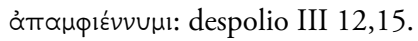

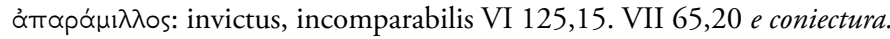

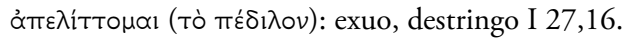

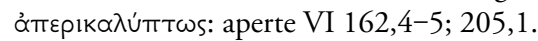

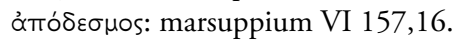

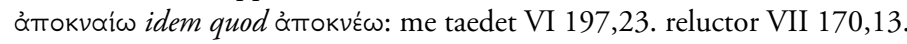




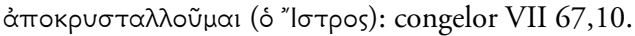

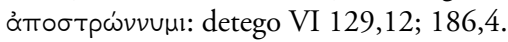

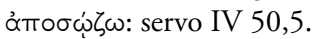

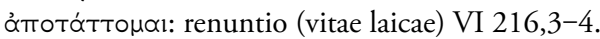

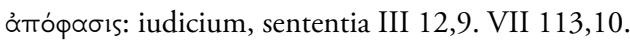

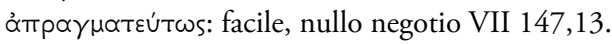

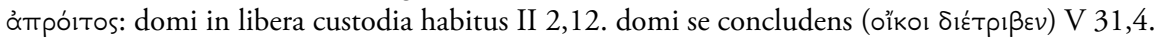

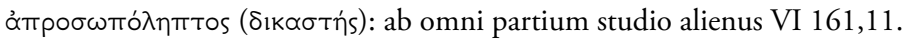

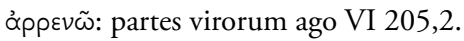

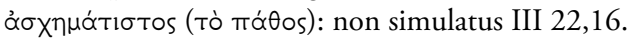

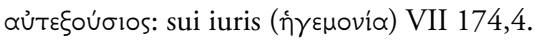

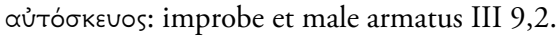

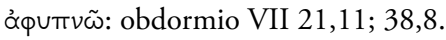

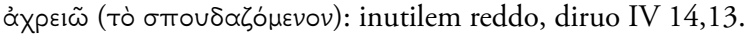

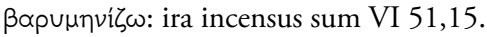

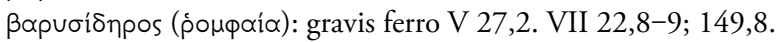

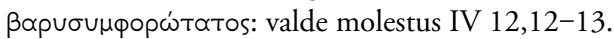

$\beta \alpha \sigma i \lambda \varepsilon i \tilde{\omega}:$ ad potestatem imperatoriam nitor I 12,4. II 2,11.

$\beta \propto \sigma \downarrow \lambda \varepsilon \cup v \omega$ : imperatorem creo VI 104,8. [imperator sum passim].

$\beta \alpha \sigma \kappa \alpha i v \omega:$ indignor VI 197,36.

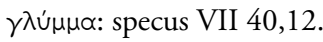

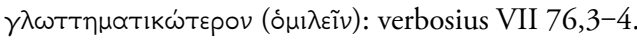

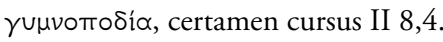

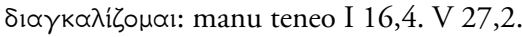

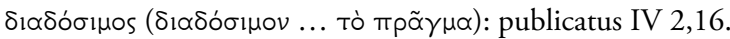

$\delta ı \alpha \theta \rho \propto \mu \beta \varepsilon v_{0} \mu \alpha$ e e coniectura : in pompa triumphali geror VI 87,14.

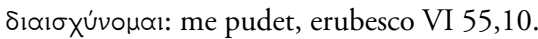

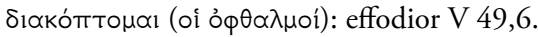

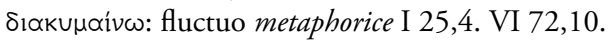

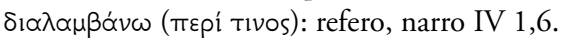

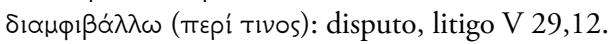

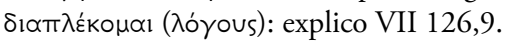

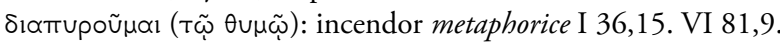

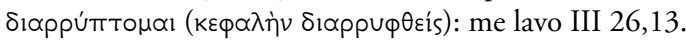

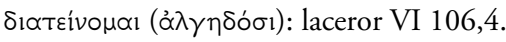

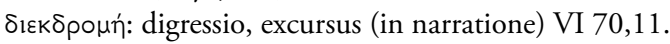

$\delta 1 \varepsilon \xi \circ 1 \delta \omega \tilde{~: ~ t u m e s c o ~ V I I ~ 51,14 . ~}$

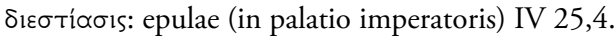

$\delta ı \varepsilon \sigma \chi \eta \mu \propto т \imath \sigma \mu \varepsilon ́ v o v$, tò: quod celatum est VI 56,3.

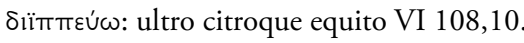

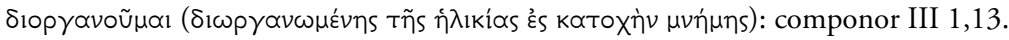

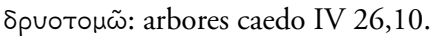

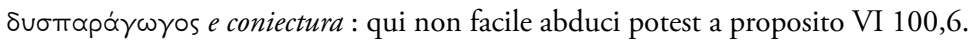

$\delta \cup \sigma \pi \alpha \rho \propto \delta \varepsilon \kappa T \tilde{\omega}:$ difficile mihi est percipere VI 41,19-20. 


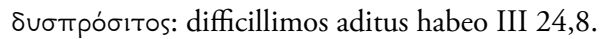

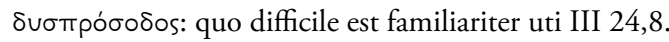

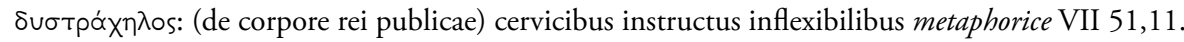

$\dot{\varepsilon} \gamma \gamma \dot{\varepsilon} v \varepsilon 1 \propto:$ status indigenarum VI 134,8.

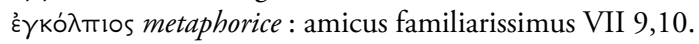

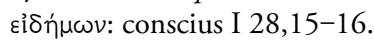

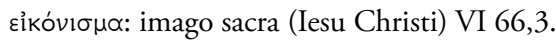

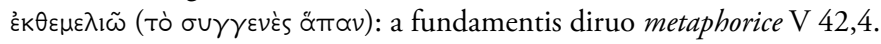

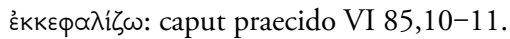

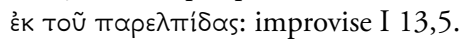

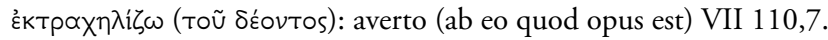

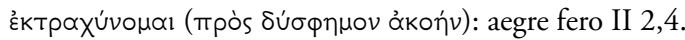

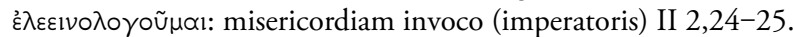

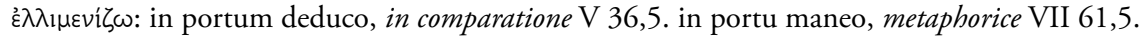

$\dot{\varepsilon} \lambda \lambda \circ \gamma ı \omega \dot{\tau} \varepsilon \rho \circ$, oi: artibus liberalibus eruditi VI 35,3.

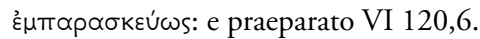

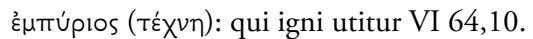

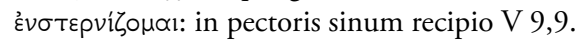

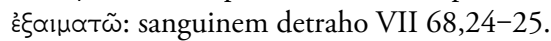

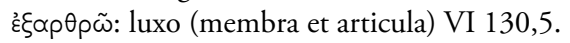

$\dot{\varepsilon} \xi \dot{\varepsilon} \pi ı \pi \varepsilon \dot{\varepsilon} \delta$ ov: de plano VI 205,5.

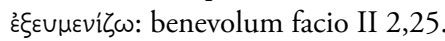

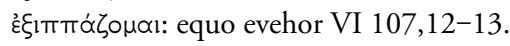

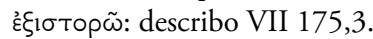

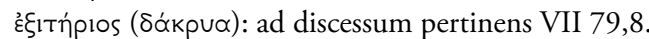

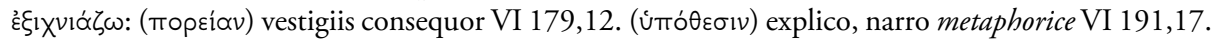

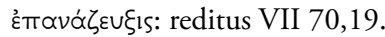

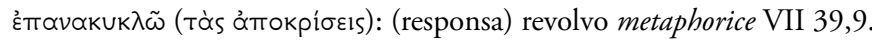

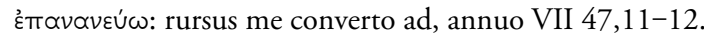

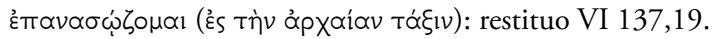

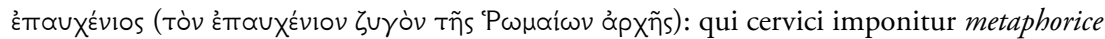

IV 40,20-21.

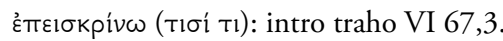

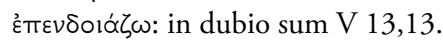

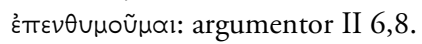

$\dot{\varepsilon} \pi 1 \theta \omega u ̈ \zeta \omega$ : vociferando incito VII 72,5; 181,2.

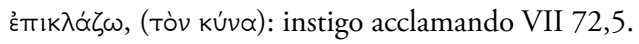

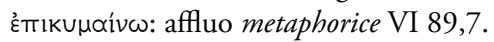

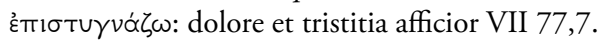

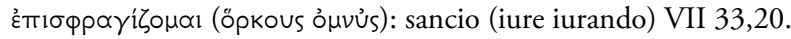

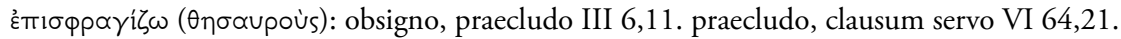

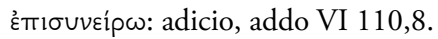

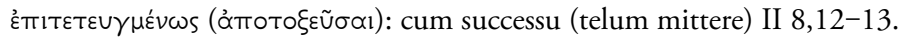

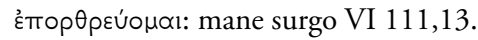

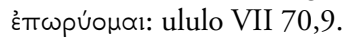

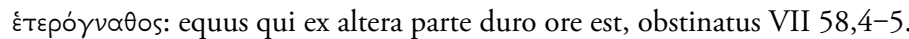




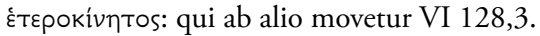

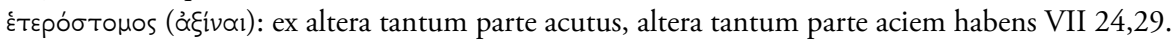

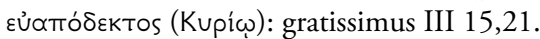

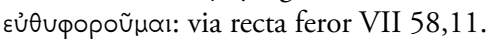

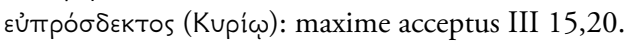

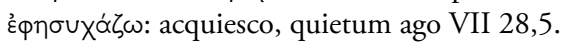

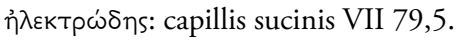

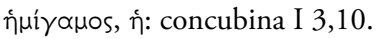

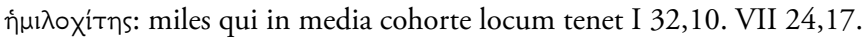

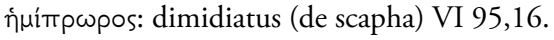

$\theta \dot{\varepsilon} \lambda \gamma \circ$ S, Tò: venustas VI 126,17.

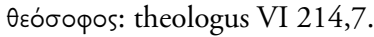

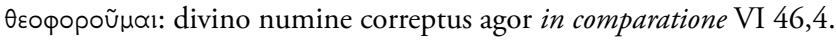

$\theta \eta \lambda \cup \pi p \varepsilon \pi \tilde{\omega}$ s: quomodo feminis convenit I 35,3.

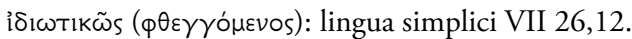

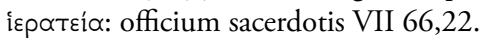

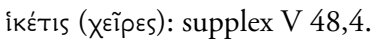

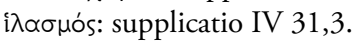

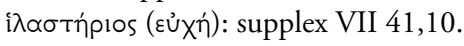

iтாள́́ $\varphi \varepsilon \sigma ı$ : dimissio equorum e carceribus II 8,3.

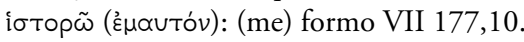

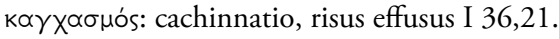

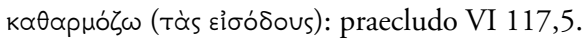

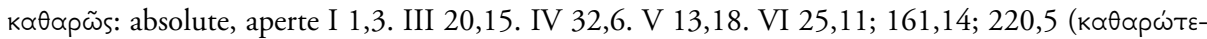
pov). VII 13,5; 42,11; 108,14; 164,11.

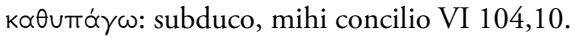

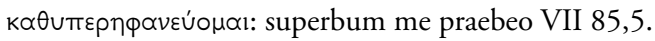

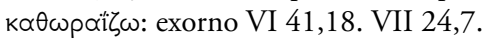

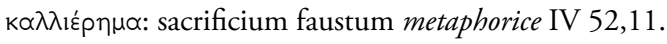

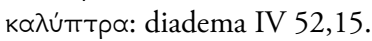

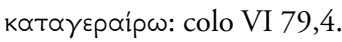

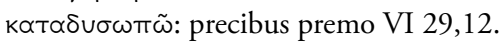

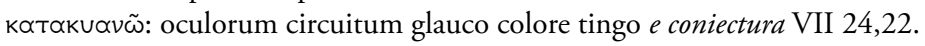

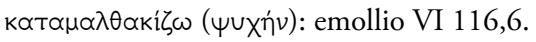

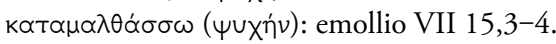

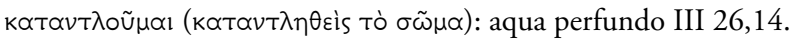

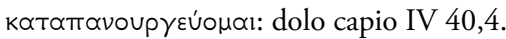

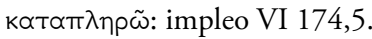

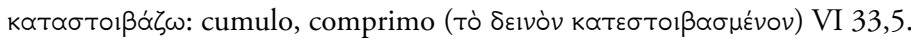

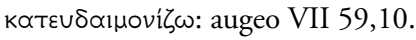

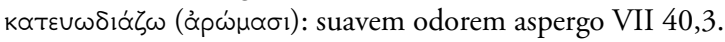

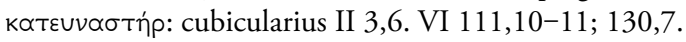

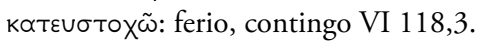




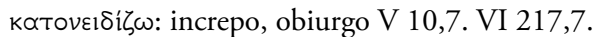

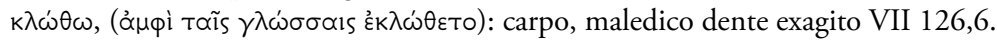

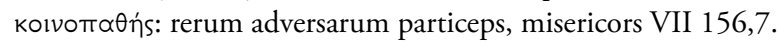

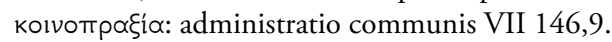

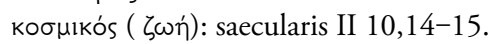

крєü $\lambda \lambda_{\text {Iov: }}$ caro corporis contemnenda VII 24,27.

кUסоґиós: tumultus III 9,22.

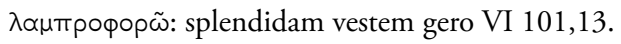

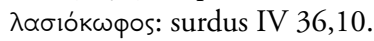

$\lambda \varepsilon 10 \kappa \cup \mu о \nu \tilde{\omega}(\theta \dot{\alpha} \lambda \alpha \sigma \sigma \alpha)$ : pacatus, levis sum VII 33,6.

$\lambda$ воитоконш̃: leonem curo metaphorice VII 18,9; 131,16.

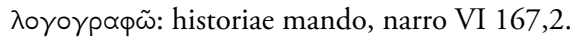

$\mu \varepsilon \gamma \propto \lambda \circ \pi \rho \varepsilon \pi \varepsilon \dot{0} \circ \mu \propto l:$ arrogantem me praebeo VII 146,4.

$\mu \varepsilon \lambda \propto \mu ф о р \tilde{\omega}:$ atra veste indutus sum, monachus sum VI 12,14.

$\mu \varepsilon \lambda \propto \nu \varepsilon 1 \mu о \nu \tilde{\omega}:$ atram vestem monachicam induo VII 162,12.

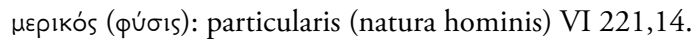

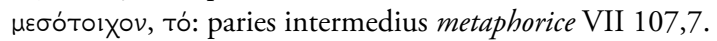

$\mu \varepsilon т \propto \kappa \cup \beta \varepsilon \cup ́ \omega:$ iterum tesseras mitto metaphorice VI 13,2.

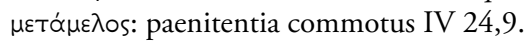

$\mu \varepsilon т \propto \mu о р ф о \tilde{\mu} \mu \propto$ : formo, recoquo V 24,10.

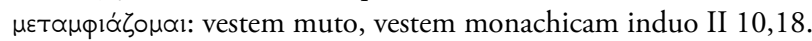

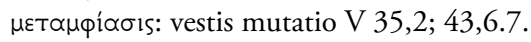

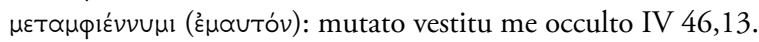

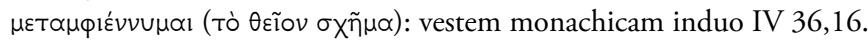

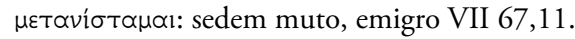

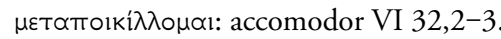

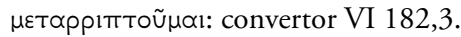

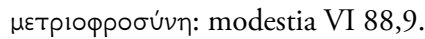

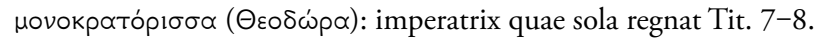

$\mu \cup \sigma \tau \eta p 1 \omega \delta \tilde{s}$ : clam IV 48,3.

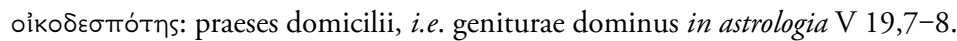

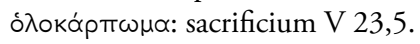

ỏ乡vypópos: qui celeriter scribit II 6,15.

ỏmtó́vouณl: aspicior II 10,21.

ỏmtáía: visio VI 96,10.

oủx' oĩov ... å $\lambda \lambda \lambda_{\alpha}$ kờ: non solum, sed etiam IV 11,13.

$\pi \alpha \mu \pi \lambda \eta \theta \varepsilon i$ : tota turba VI 85,4 . VII 139,10

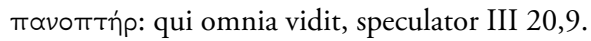

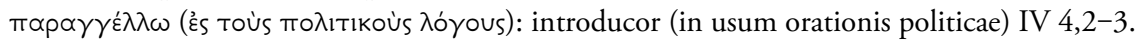

$\pi \propto p \propto \delta 101 k \tilde{\omega}$ : secundum locum teneo administrationis I 19,7. VI 179,11. VII 33,14-15.

$\pi \propto p \propto \delta \circ \xi о \pi о เ \tilde{:}$ : miraculum perficio VI 183,10. VII 115,6-7.

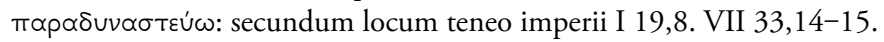

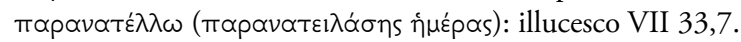




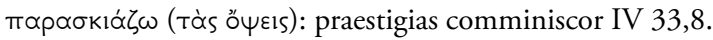

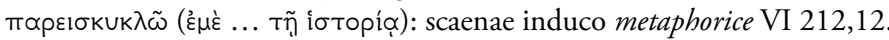

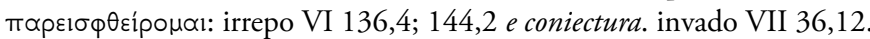

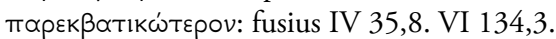

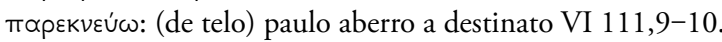

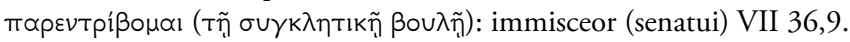

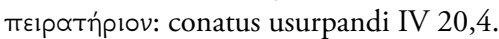

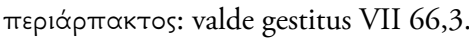

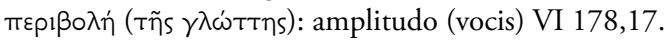

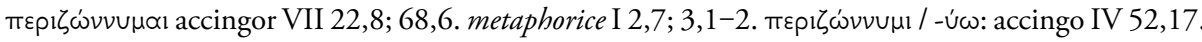

VII 122,4. circumsisto VI 94,11; 107,8.

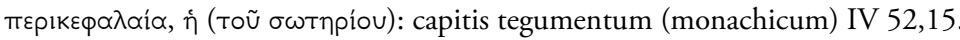

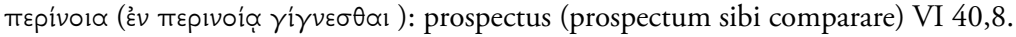

$\pi \varepsilon p 1 o p \circ \propto \tilde{\omega}$ : tectum formo (hastis) VII 24,31.

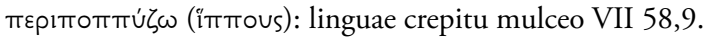

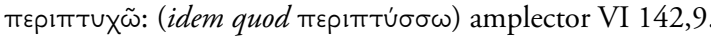

$\pi \varepsilon \rho ı$

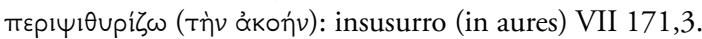

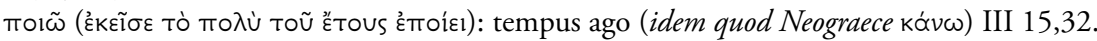

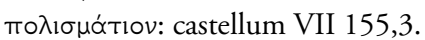

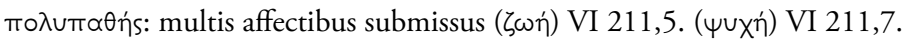

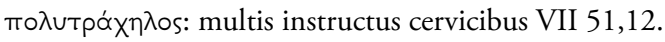

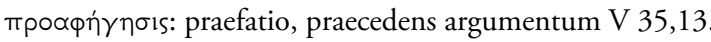

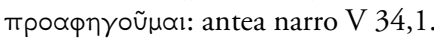

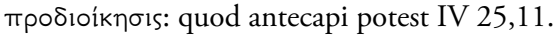

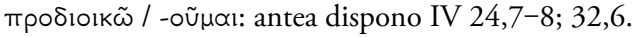

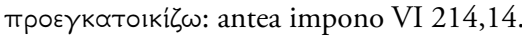

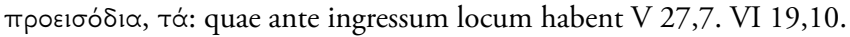

$\pi \rho \circ \theta \varepsilon \rho \pi \varepsilon \varepsilon \dot{v} \omega:$ antea colo V 8,4.

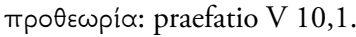

троїбтори̃: antea narro VI 71,4.

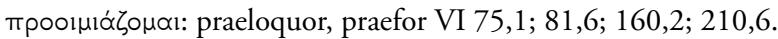

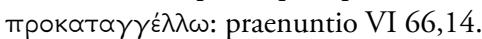

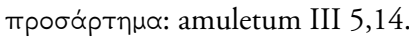

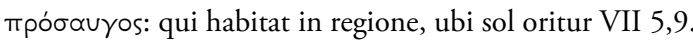

$\pi \rho \circ \sigma \beta ı \beta \alpha ́ \zeta \omega ~\left(\tau \tilde{\omega} \lambda{ }^{\prime} \gamma \omega\right)$ : adicio, addo e coniectura VII 103,2.

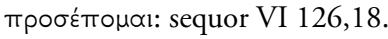

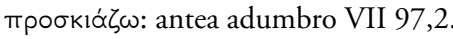

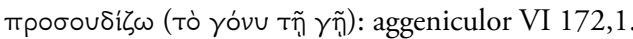

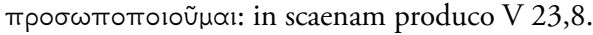

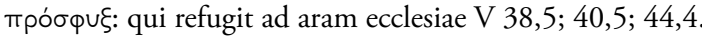

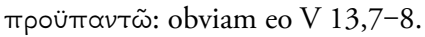

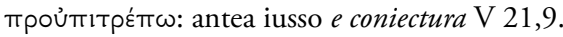

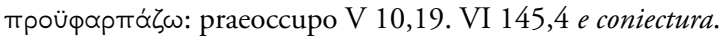

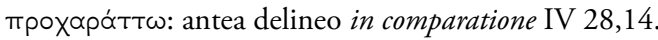

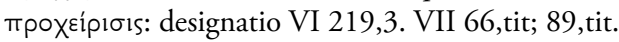

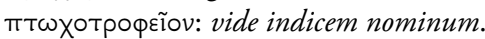




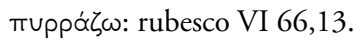

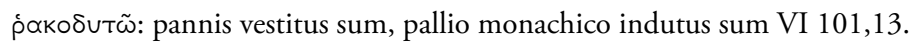

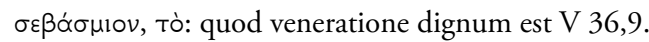

бıбupoфópos, ó: qui vestem e pellibus caprinis consutam gestat VI 134,11.

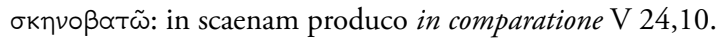

бкпuoupyós: actor, scaenicus VI 141,8.

бKı $\gamma \rho \propto \phi \tilde{:}$ : adumbro, paucis expono VII 165,2.

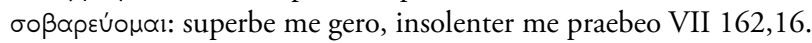

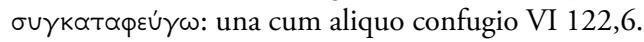

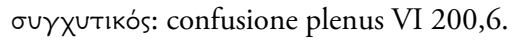

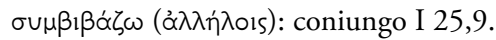

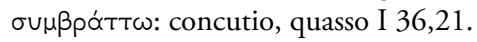

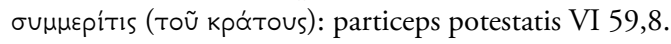

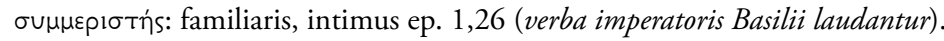

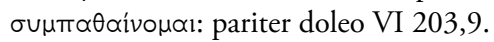

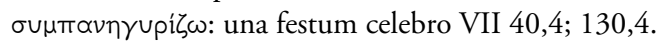

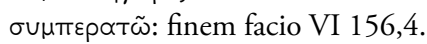

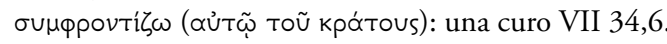

$\sigma u v \propto \gamma \kappa \propto \lambda i \zeta \zeta o \mu \propto l:$ amplector VII 16,6.

бuvóveıul: concurro VII 149,11.

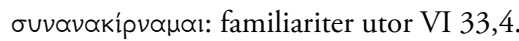

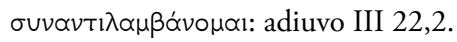

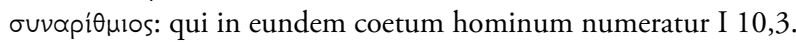

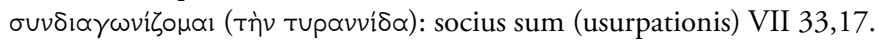

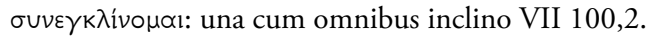

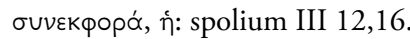

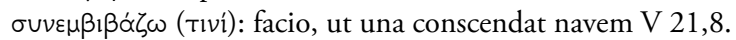

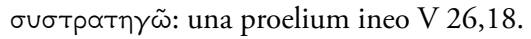

$\sigma u v \varepsilon \xi \alpha \phi \propto v i \zeta \zeta \omega(\dot{\varepsilon} \lambda \pi \pi i \delta \propto s):$ omnino deleo V 2,14.

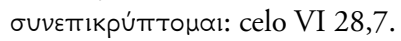

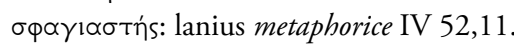

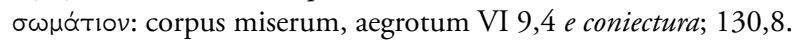

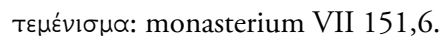

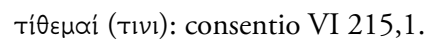

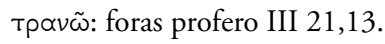

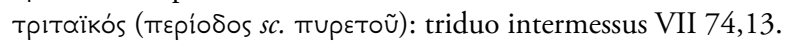

трıттús: tertius (= tertium gradum dignitatis in senatu tenens) VI 3,11.

úßpıтா $\alpha \tilde{\omega}$ : iniuriam patior VII 156,8.

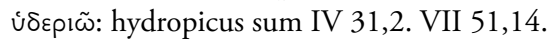

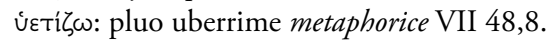

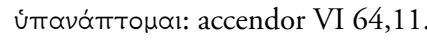

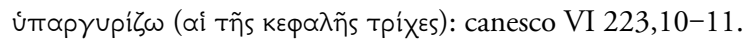

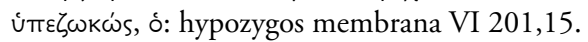




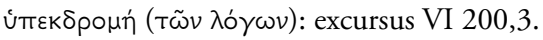

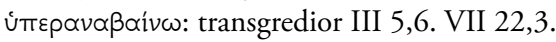

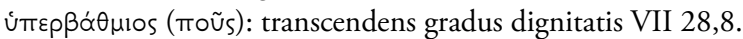

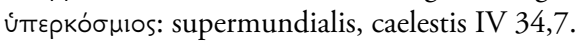

Úтr

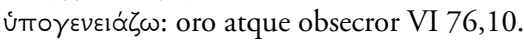

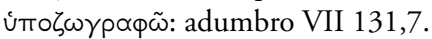

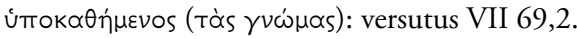

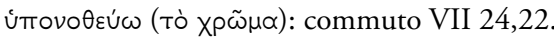

(ن்

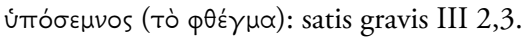

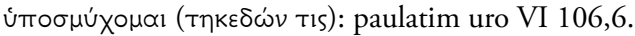

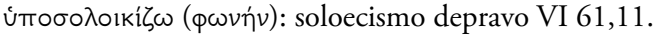

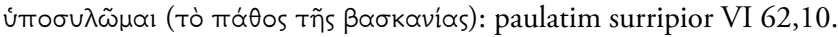

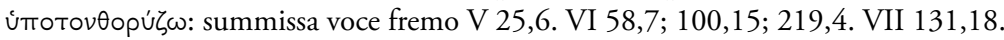

фpovтıбтńplov: monasterium I 20,17. IV 52,7. VII 164,9.

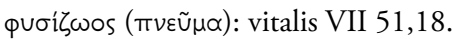

$\chi \propto \lambda_{1 v} \propto \gamma \omega \gamma \tilde{\omega}:$ loro duco metaphorice VII 174,3.

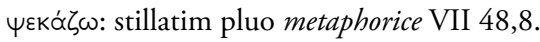

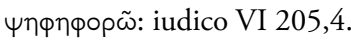

